\title{
Cost Effectiveness of PD-L1-Based Test-and-Treat Strategy with Pembrolizumab as the First-Line Treatment for Metastatic NSCLC in Hong Kong
}

\author{
Herbert H. Loong ${ }^{1} \cdot$ Carlos K. H. Wong ${ }^{2}$ - Linda Kam Suet Leung ${ }^{1} \cdot$ Praveen Dhankhar $^{3} \cdot$ Ralph P. Insinga $^{4} \cdot$ \\ Sheenu Chandwani ${ }^{4}$. Danny C. Hsu ${ }^{5}$ Mary Y. K. Lee ${ }^{5}$. Min Huang ${ }^{4} \cdot$ James Pellissier ${ }^{4}$. Akanksha Rai ${ }^{3}$. \\ Monika Achra ${ }^{3}$. Seng Chuen Tan ${ }^{6}$
}

Published online: 17 September 2019

(c) The Author(s) 2019

\begin{abstract}
Background Pembrolizumab, a monoclonal antibody against programmed death ligand 1 (PD-L1), is approved by several regulatory agencies for first-line treatment of metastatic non-small-cell lung cancer (NSCLC) with a PD-L1 tumor proportion score (TPS) $\geq 50 \%$ and no epidermal growth factor receptor (EGFR) or anaplastic lymphoma kinase genomic tumor aberrations. This study was conducted from the perspective of the Hospital Authority in Hong Kong and aimed to evaluate the cost effectiveness of a biomarker (PD-L1) test-and-treat strategy (BTS), in which patients with a TPS $\geq 50 \%$ received pembrolizumab and other patients received platinum doublet chemotherapy versus all patients receiving platinum doublet chemotherapy.

Methods The model used a partitioned survival approach to estimate the incremental cost-effectiveness ratio (ICER) expressed as the cost per quality-adjusted life-year (QALY) gained. The clinical efficacy, utility and safety data were derived from the KN024 trial. Costs and health outcomes were projected over a 10-year time horizon and discounted at 3\% per year. Costs for drug acquisition, PD-L1 testing, drug administration and disease management were used. Sensitivity analyses were conducted to evaluate the robustness of results.

Results The BTS approach led to an increase of 0.29 QALYs at an additional cost of Hong Kong dollars (HK\$) 249,077 (US\$31,933) compared with platinum doublet chemotherapy, resulting in an ICER of HK\$865,189 (US\$110,922) per QALY gained. This is lower than the World Health Organization cost-effectiveness threshold of three times the 2016 gross domestic product (GDP) per capita for Hong Kong of HK\$1017,819 (US\$130,490). Probabilistic sensitivity analyses showed a 59.4\% chance that the ICER would be below this threshold.

Conclusion First-line treatment with pembrolizumab in a BTS to identify patients with NSCLC with PD-L1 TPS $\geq 50 \%$ can be considered cost effective in Hong Kong compared with platinum doublet chemotherapy based on a three-times GDP per capita threshold. However, local data on clinical efficacy and safety were not available to estimate overall survival (OS) and progression-free survival (PFS) specific to patients with NSCLC in Hong Kong. Further, uncertainty is inherent in the survival projections/extrapolation of PFS and OS beyond the trial period, and future research may help to further inform these parameters.
\end{abstract}

Electronic supplementary material The online version of this article (https://doi.org/10.1007/s41669-019-00178-7) contains supplementary material, which is available to authorized users.

Ralph P. Insinga

ralph_insinga@merck.com

1 Department of Clinical Oncology, State Key Laboratory in Oncology of South China, The Chinese University of Hong Kong, Hong Kong, Hong Kong

2 Department of Family Medicine and Primary Care, The University of Hong Kong, Hong Kong, Hong Kong
3 Complete HEOR Solutions LLC, 1120 Welsh Road, Suite 205, North Wales, PA 19454, USA

4 Merck Sharp and Dohme Corp, Center for Observational and Real-World Evidence, North Wales, PA, USA

5 Merck Sharp \& Dohme (Asia) Ltd, Hong Kong, Hong Kong

6 Merck Sharp \& Dohme (Asia Pacific), Singapore, Singapore 


\section{Key Points for Decision Makers}

A programmed death ligand 1 (PD-L1) test-and-treat strategy for the use of pembrolizumab in patients undergoing first-line treatment for non-small-cell lung cancer (NSCLC) with PD-L1 expression $\geq 50 \%$ is associated with a gain of 0.29 quality-adjusted life-years (QALYs), at an additional total cost of Hong Kong dollars (HK\$) 249,077 per patient compared with platinum doublet chemotherapy (incremental cost-effectiveness ratio [ICER] of HK\$865,189 per QALY gained) (year 2016 values).

Key cost-effectiveness drivers were the drug acquisition costs and projections of improved survival.

The ICER was below the threshold of three times gross domestic product per capita for Hong Kong.

\section{Introduction}

With approximately 1.5 million cases diagnosed globally each year, lung cancer is the most common malignancy [1] and is responsible for nearly one in five cancer-related deaths [2]. In Hong Kong, lung cancer is the most common cancer in men and third most common among women, with age-standardized incidence rates of 47.1 and 25.6 per 100,000 individuals, respectively. According to the Hong Kong Cancer Registry, in 2015 there were 4748 new cases of lung cancer diagnosed (i.e., $15.7 \%$ of all incident cancers) and 4031 deaths $(28.2 \%$ of all cancer deaths) [3, 4]. Nonsmall-cell lung cancer (NSCLC) accounts for $85-90 \%$ of all lung cancer cases worldwide [1,5]. Long-term prognosis for patients with NSCLC is poor, as $50-60 \%$ of patients present with advanced-stage disease at diagnosis $[1,6]$. This places substantial economic burden on healthcare systems. During 2015, for example, lung cancer accounted for $13.1 \%$ of hospitalizations due to all cancer types in Hong Kong [4].

Platinum-based chemotherapy (with or without maintenance therapy) and subsequent follow-up with second-line cytotoxic chemotherapy is the standard of care for most patients with advanced NSCLC, which leads to a median survival of approximately 1 year [7-9]. Immunotherapy is emerging as a new oncology paradigm for NSCLC treatment, which targets the programmed death ligand 1 (PDL1) pathway. Pembrolizumab is a highly selective, humanized monoclonal antibody against PD-L1 that inhibits the complex interaction of PD-L1 with PD-L1/PD-L2 receptors, thereby activating the expression of $\mathrm{T}$ cells and releasing PD-L1-mediated inhibition of the immune response, including the antitumor immune response. Pembrolizumab has increased activity in tumors that express PD-L1 in advanced NSCLC [10]. The US FDA approved pembrolizumab in October 2016 for first-line treatment in metastatic NSCLC with a PD-L1 tumor proportion score (TPS) $\geq 50 \%$ as determined by an FDA-approved test, with no epidermal growth factor receptor (EGFR) or anaplastic lymphoma kinase (ALK) genomic tumor aberrations [11]. This indication has also been approved by several other regulatory agencies (e.g., the European Medicines Agency, the UK Medicines and Healthcare Products Regulatory Agency and Health Canada) $[12,13]$. In Hong Kong, pembrolizumab has been registered for first-line treatment of metastatic NSCLC with high PD-L1 expression (TPS $\geq 50 \%$ ) since September 2017 according to the latest Hong Kong package insert. Regulatory approval of pembrolizumab in this indication was obtained in different countries following demonstration of improved overall survival (OS) and progression-free survival (PFS) versus chemotherapy within the KEYNOTE-024 (KN024) trial. The KN024 trial was an open-label, randomized, phase III trial that compared pembrolizumab (fixed dose of $200 \mathrm{mg}$ every 3 weeks) with the investigator's choice of platinum-based chemotherapy as first-line therapy in patients with metastatic NSCLC and a PD-L1 TPS $\geq 50 \%$. The trial demonstrated that patients treated with pembrolizumab had superior OS compared with those treated with platinum doublet chemotherapy (hazard ratio [HR] for mortality $0.60 ; 95 \%$ confidence interval [CI] $0.41-0.89 ; p=0.005)$. PFS was also significantly longer with pembrolizumab than with platinum doublet chemotherapy (HR 0.50; 95\% CI 0.37-0.68; $p<0.001$ ). Pembrolizumab was less commonly associated with grade 3-5 treatmentrelated adverse events (AEs) than was platinum doublet chemotherapy, indicating its relative safety and tolerability. The characteristics of the simulated cohort in KN024 are briefly described in the Electronic Supplementary Material (ESM; file 3), and the full details of the KN024 trial are described elsewhere [13].

Given the rising epidemiological and economic burden of NSCLC in Hong Kong, evaluating the clinical effectiveness and the cost effectiveness of a new therapy is a prerequisite for enabling patient access to any new intervention [15]. We conducted a cost-effectiveness analysis of a biomarker test-and-treat strategy (BTS) with pembrolizumab versus platinum doublet chemotherapy from the perspective of the Hong Kong Hospital Authority. A threshold for establishing cost effectiveness is based on a World Health Organization (WHO) recommendation of three times the gross domestic product (GDP) per capita; for Hong Kong, this is equivalent to Hong Kong dollars (HK\$) 1017,819 (US\$130,490) for 2016 [43, 44].

The objective of this study was to compare the costs and benefits of employing a PD-L1 BTS. In this BTS, patients received a PD-L1 test, and those with a TPS $\geq 50 \%$ 
(prevalence of $30.2 \%$ ) received pembrolizumab; the other patients received platinum doublet chemotherapy [13]. This approach was compared against a strategy in which patients did not receive a PD-L1 test and were treated with platinum doublet chemotherapy. We also examined a scenario to assess the cost effectiveness in high expressers (see file 2 in the ESM for details).

\section{Methods}

\subsection{Model Overview}

We developed a partitioned survival model [16] to evaluate the expected costs and outcomes for pembrolizumab compared with platinum doublet chemotherapy as first-line therapy in patients with NSCLC. The incremental cost per quality-adjusted life-year (QALY) gained and incremental cost per life-year (LY) gained were used as the main outcome measures to assess cost effectiveness. Clinical efficacy, utility and safety data were derived from the KN024 trial, with a data cut-off date of 9 May 2016. This phase III randomized controlled trial compared pembrolizumab with the investigator's choice among five platinum-based regimens in first-line treatment of metastatic NSCLC [14].

In line with recommendations from several health technology assessment agencies (e.g., the UK National Institute for Health and Care Excellence (NICE) [17] and the Canadian Agency for Drugs and Technologies in Health (CADTH) [18]), we used a 10-year time horizon in the base case to adequately capture differences in health effects and healthcare resource use between the interventions. Costs and health outcomes were discounted at a rate of $3 \%$ per year [19].

\subsection{Model Structure}

We used a cohort simulation model (Fig. 1) to evaluate the outcomes and costs associated with each regimen. The model included three health states: progression free (PF), progressive disease (PD) and death. The simulation model combined the observed OS curve during the trial with a statistical extrapolation to project beyond the KN024 study period.

A model cycle length of 1 week was used to accommodate different schedules of platinum doublet chemotherapy. A half-cycle correction was used to improve the accuracy of the results.

To evaluate the BTS approach, we assumed that the efficacy of platinum doublet chemotherapy derived from KN024 reflected the efficacy of platinum doublet chemotherapy in all patients undergoing first-line therapy for metastatic NSCLC, irrespective of PD-L1 expression [20, 21].

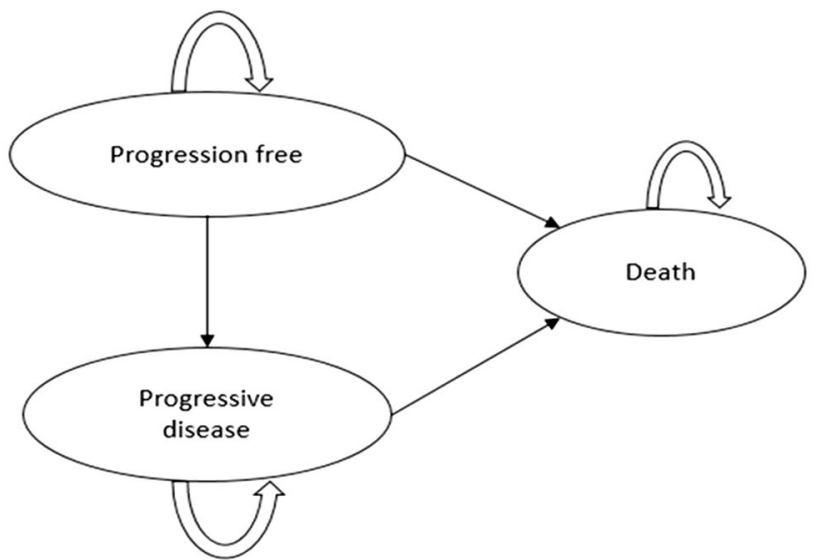

Fig. 1 Transition diagram of the cohort simulation model to estimate health economic outcomes

\subsection{Interventions}

In the BTS approach, patients with strong positive PD-L1 expression (TPS $\geq 50 \%$ ) (30.25\% of all patients) were treated with pembrolizumab; all other patients received platinum-based chemotherapy. To be treated with pembrolizumab, patients must have had a PD-L1 test done with the result indicating a TPS $\geq 50 \%$ for first-line pembrolizumab monotherapy use. Currently, only one commercially available PD-L1 test is validated for pembrolizumab: the PD-L1 IHC 22C3 pharmDx. Each kit contains reagents to perform 50 tests in up to 15 individual runs. Each test costs HK\$1700 based on Hong Kong Hospital Authority costs.

In KN024, patients were randomly assigned, in a 1:1 ratio, to receive treatment with either pembrolizumab for up to 35 cycles or the platinum-based chemotherapy regimens for 4 to 6 cycles (Table 1). Patients with non-squamous disease who received pemetrexed combination therapy could continue to receive pemetrexed as maintenance therapy after the completion of combination chemotherapy [13]. The comparator, platinum-based chemotherapy, was considered standard of care in Hong Kong before pembrolizumab became available.

\subsection{Efficacy Inputs}

\subsubsection{Clinical Effectiveness}

As per the protocol, treatment was continued for the specified number of cycles or until disease progression, intolerance, patient withdrawal or investigator decision. Patients receiving platinum doublet chemotherapy who had documented disease progression could cross over to receive pembrolizumab [13]. Clinically stable patients considered 
Table 1 Platinum-based chemotherapy regimens

\begin{tabular}{|c|c|c|c|c|c|c|c|}
\hline Regimen & Therapy & Dose $\left(\mathrm{mg} / \mathrm{m}^{2}\right)$ & Frequency & $\begin{array}{l}\text { Duration } \\
\text { (cycles) }\end{array}$ & $\begin{array}{l}\text { Distribu- } \\
\text { tion }(\%)\end{array}$ & Vial size $(\mathrm{mg})$ & Vial price $(\mathrm{HK} \$)$ \\
\hline \multirow[t]{2}{*}{$1^{\mathrm{a}}$} & Carboplatin & 300 & $\begin{array}{l}\text { Day } 1 \text { of every } \\
\text { 3-week cycle }\end{array}$ & $4-6$ & 44 & 450 & 239.38 \\
\hline & Pemetrexed & 500 & $\begin{array}{l}\text { Day } 1 \text { of every } \\
\text { 3-week cycle }\end{array}$ & $4-6^{\mathrm{b}}$ & & $\begin{array}{l}100 \\
500\end{array}$ & $\begin{array}{l}2220 \\
9000\end{array}$ \\
\hline \multirow[t]{2}{*}{$2^{\mathrm{a}}$} & Cisplatin & 75 & $\begin{array}{l}\text { Day } 1 \text { of every } \\
\text { 3-week cycle }\end{array}$ & $4-6$ & 24 & 50 & 98.88 \\
\hline & Pemetrexed & 500 & $\begin{array}{l}\text { Day } 1 \text { of every } \\
\text { 3-week cycle }\end{array}$ & $4-6^{\mathrm{b}}$ & & $\begin{array}{l}100 \\
500\end{array}$ & $\begin{array}{l}2220 \\
9000\end{array}$ \\
\hline \multirow[t]{2}{*}{3} & Carboplatin & 300 & $\begin{array}{l}\text { Day } 1 \text { of every } \\
3 \text {-week cycle }\end{array}$ & $4-6$ & 13 & 450 & 239.38 \\
\hline & Gemcitabine & 1000 & Days 1 and 8 of every 3 -week cycle & $4-6$ & & $\begin{array}{r}200 \\
1000\end{array}$ & $\begin{array}{l}51.56 \\
194.13\end{array}$ \\
\hline \multirow[t]{2}{*}{4} & Cisplatin & 75 & $\begin{array}{l}\text { Day } 1 \text { of every } \\
3 \text {-week cycle }\end{array}$ & $4-6$ & 7 & 50 & 98.88 \\
\hline & Gemcitabine & 1000 & Day 1 and 8 of every 3 -week cycle & $4-6$ & & $\begin{array}{r}200 \\
1000\end{array}$ & $\begin{array}{l}51.56 \\
194.13\end{array}$ \\
\hline \multirow[t]{2}{*}{5} & Carboplatin & 300 & $\begin{array}{l}\text { Day } 1 \text { of every } \\
\text { 3-week cycle }\end{array}$ & $4-6$ & 11 & 450 & 239.38 \\
\hline & Paclitaxel & 175 & $\begin{array}{l}\text { Day } 1 \text { of every } \\
\text { 3-week cycle }\end{array}$ & $4-6^{\mathrm{c}}$ & & $\begin{array}{r}30 \\
100\end{array}$ & $\begin{array}{l}70.70 \\
200.99\end{array}$ \\
\hline
\end{tabular}

$H K \$$ Hong Kong dollar

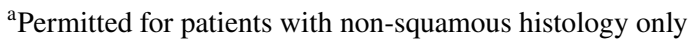

${ }^{b}$ Followed by optional pemetrexed maintenance therapy given at a dose of $500 \mathrm{mg} / \mathrm{m}^{2}$ every 3 weeks

${ }^{c}$ Followed by optional pemetrexed maintenance therapy given at a dose of $500 \mathrm{mg} / \mathrm{m}^{2}$ every 3 weeks (patients with non-squamous histology only)

to be deriving clinical benefit were eligible to continue pembrolizumab beyond progression. Patients treated with pembrolizumab were assumed to be treated for a maximum of 2 years ( 35 cycles) for the initial course as per trial protocol and FDA-approved label; patients with disease progression were eligible for re-treatment of 1 year after completing the initial course [14, 22].

Effectiveness data, including PFS and OS, were estimated from KN024. Standard parametric models were fitted to these data to extrapolate the outcomes as per NICE Decision Support Unit guidelines [23]. The proportional hazard assumption was verified to determine whether independent survival models were to be explored in each treatment arm. The standard parametric models were fitted using exponential, Weibull, Gompertz, Log-logistic, Log-normal and generalized Gamma distributions. Visual inspection, along with the Akaike information criterion (AIC) and the Bayesian information criterion (BIC) goodness-of-fit statistics, were used to assess the best-fitting parametric distributions. The clinical plausibility of the extrapolated results was also considered when selecting the distributional functions for the model.

\subsubsection{Time-on-Treatment}

In Hong Kong, patients pay either the partial or the full costs of new anticancer drugs, and treatment is generally stopped when disease progression is documented. Hence, in the model, treatment duration was assumed to be the same as PFS for the base-case analysis.

In clinical practice, pembrolizumab treatment or pemetrexed maintenance in platinum-based chemotherapy is expected to be continued until documented disease progression, unacceptable AEs or patient/physician decision to interrupt treatment. In the KN024 protocol, when none of these criteria were met, a stopping rule of 2 years was determined. In the model, the expected number of treatment cycles per patient was based on the estimated time on treatment, up to a maximum of 2 years.

\subsubsection{Progression-Free Survival}

To model PFS, the Kaplan-Meier (KM) curve was used directly for the first 9 weeks, and parametric functions were then used to project survival over the time horizon of 
interest. The cut-off time of 9 weeks was chosen as it coincided with the first radiologic tumor response assessment performed per the trial protocol. Weibull and exponential distributions were selected as the best fit for pembrolizumab and platinum doublet chemotherapy, respectively, as per the $\mathrm{AIC}$ and BIC and visual inspection criterion.

\subsubsection{Overall Survival}

In KN024, a total of $66(43.7 \%)$ patients in the platinum doublet chemotherapy arm switched to receive pembrolizumab after documented disease progression, which potentially diluted the survival benefits associated with pembrolizumab. A simplified two-stage approach as described by Latimer and Abrams [24] and Latimer [25] was implemented to perform an adjusted OS analysis to correct for the potential bias induced by treatment switch in the platinum doublet chemotherapy arm. We modeled the OS for platinum doublet chemotherapy based on the trial data, both with and without switching adjustment. In the base-case analysis, we used the data without switching adjustment, as it reflects the current reality that PD-L1 inhibitors are available in Hong Kong for the second-line treatment of PD-L1-positive NSCLC.

For the OS outcomes, the standard parametric curves did not provide a good visual fit for the observed KM data, so we performed a two-phase piecewise extrapolation. Using Chow tests [26, 27], the cut-off points for the piecewise extrapolation were obtained (week 32 for pembrolizumab, week 38 for platinum doublet chemotherapy unadjusted). The KM curve was used directly for the first phase until the cut-off, whereas an exponential curve was fitted to the remaining $\mathrm{KM}$ data to project OS, as selected based on AIC, BIC and visual inspection. The area under the resulting piecewise curve was used to estimate OS.

\subsubsection{Other Clinical Inputs and Subsequent Therapy}

Clinical inputs such as AE incidence rates (grade 3-5), weight and body surface area (BSA) distribution were estimated from KN024. AEs that were reported in $\geq 5 \%$ of patients in either arm (i.e., pembrolizumab or platinum doublet chemotherapy) were included. Pneumonitis, although reported in $<5 \%$ of patients in both arms, was also included per clinicians' advice. Based on incidences of grade $3+$ AEs from KN024, and management costs from public hospitals in Hong Kong, the total average cost per patient for managing AEs was reported.

Time to disease progression was used as a proxy for time on first-line treatment. Once progression occurred, patients received subsequent lines of treatment according to the probabilities and regimens described in Table 2. A one-time cost of subsequent therapy for each treatment arm was also included in the analysis. This cost was derived based on the distribution of patients receiving each subsequent drug regimen in KN024, list prices and average treatment duration (e.g., 183 days for pembrolizumab). These subsequent

Table 2 Distribution of post-discontinuation therapy in the model

\begin{tabular}{|c|c|c|c|}
\hline Post-discontinuation regimen & Pembrolizumab & $\begin{array}{l}\text { Chemotherapy ITT without } \\
\text { adjustment }\end{array}$ & $\begin{array}{l}\text { Chemotherapy with } \\
\text { switching adjust- } \\
\text { ment }\end{array}$ \\
\hline$\%$ receiving subsequent therapy in discontinued patients & 43.75 & 59.26 & 59.26 \\
\hline$\%$ receiving second-line therapy & 100.00 & 100.00 & 100.00 \\
\hline \multicolumn{4}{|l|}{ Distribution of second-line therapy } \\
\hline Carboplatin + gemcitabine & 16.67 & & \\
\hline Carboplatin + paclitaxel + bevacizumab & 16.67 & & \\
\hline Carboplatin + pemetrexed & 45.83 & & \\
\hline Cisplatin + pemetrexed & 20.83 & & \\
\hline Docetaxel & & 1.30 & 33.33 \\
\hline Nivolumab & & 6.49 & \\
\hline Pembrolizumab & & 89.61 & \\
\hline Pemetrexed & & 2.60 & 66.67 \\
\hline$\%$ receiving second-line maintenance therapy & 25.71 & 0.00 & 0.00 \\
\hline \multicolumn{4}{|l|}{ Distribution of second-line maintenance therapy } \\
\hline Pemetrexed & 100.00 & & \\
\hline$\%$ receiving third-line therapy & 5.71 & 15.00 & 15.00 \\
\hline \multicolumn{4}{|l|}{ Distribution of third-line therapy } \\
\hline Docetaxel & 100.00 & 100.00 & 100.00 \\
\hline
\end{tabular}

Data are presented as percentages

ITT intention to treat 
therapy costs and AE costs for each treatment arm are provided in Table 3.

\subsection{Utilities}

Two approaches for utilities were used to estimate qualityadjusted survival time in the model: utility by time to death and by health states (PF, PD). In the base case, we used time to death as the primary approach to measure utilities, which reflects the decline in cancer patients' quality of life as they approach death. It uses more health states and allows for a more refined modeling of decline in health-related quality of life for patients as they approach death (e.g., for patients in the model, dying of causes unrelated to disease progression or patients who may respond to subsequent therapy postprogression with remission or cure). Utilities were collected in KN024 using the EuroQol 5-Dimensions, 3 levels (EQ5D-3L) instrument. Scores from the pooled treatment groups were used because there were no statistically significant differences in EQ-5D scores in each time-to-death category between the treatment arms [28]. We converted the EQ-5D responses to population-based utility values using published scoring algorithms. For the base-case analysis, a US-based scoring algorithm was applied to US patients [29], whereas the UK-based algorithm was applied to UK patients [30] and the EU-based algorithm was applied to all other patients [31].

\subsection{Costs}

The cost inputs included in this analysis were PD-L1 test cost, regimen-related costs (including drug acquisition, administration and premedication), non-drug-related disease management costs (see file 1 in the ESM for details), subsequent therapy costs, terminal care costs and AE management costs (see Table 4). All costs prior to 2016 were updated to HK\$, year 2016 values.

Pembrolizumab was administered at a fixed dose of $200 \mathrm{mg}$ every 3 weeks. Assuming no co-insurance or copayment, the average cost per dose of pembrolizumab was based on the 2017 list price of HK\$58,628 (US\$7516) for two $100-\mathrm{mg}$ vials.

Table 3 Other costs considered in the model

\begin{tabular}{lc}
\hline Cost item & Costs (HK\$) \\
\hline Subsequent therapy cost & \\
Pembrolizumab arm & 60,740 \\
Platinum doublet chemotherapy arm & 292,794 \\
Adverse event management cost & \\
Pembrolizumab arm & 2985 \\
Platinum doublet chemotherapy arm & 7357 \\
\hline
\end{tabular}

$H K \$$ Hong Kong dollar
The average number of vials of platinum doublet chemotherapy regimens was calculated using the BSA distribution and the weight of patients with metastatic NSCLC (Table 4). The platinum doublet chemotherapy cost of HK\$12,599 (US\$1615) per dose was calculated based on the distribution of each regimen in KN024. The calculated cost per dose of pemetrexed maintenance was HK\$17,603 (US\$2257). Under the assumption of no vial sharing, vial wastage was modeled for platinum doublet chemotherapy and maintenance but not for pembrolizumab given the fixed dosage.

\subsection{Sensitivity Analyses}

We performed a series of one-way deterministic sensitivity analyses (DSA) and a probabilistic sensitivity analysis (PSA) to examine the model robustness to reasonable changes in key model parameters and assumptions. The key parameters investigated related to PFS, OS and time on treatment, utilities and costs (disease management costs, AE costs, subsequent therapy costs and terminal care costs).

The PSA simulation included 1000 iterations, in which a different set of values for each of the key model parameters was randomly drawn from their respective probability distributions (file 4 in the ESM). The resulting 1000 estimates for the ICER were displayed using a scatterplot and a costeffectiveness acceptability curve (CEAC).

Several additional scenarios were also explored and are reported in file 2 in the ESM. These included the impact of a treatment-switching adjustment. The other sensitivity analyses adjusted the model time horizon, discounting of costs and outcomes and application of alternative plausible parametric functions to the extrapolation of OS and PFS. Results were also obtained varying the price of pembrolizumab and the utility algorithm and estimation approach.

\subsection{Model Validation}

The model described herein represents an adaptation for Hong Kong of a previously published US cost-effectiveness model [14]. Health economists, clinicians and pharmacy professionals who are experts in oncology evaluated the base US model. Detailed review and feedback were also provided by an expert panel of three medical oncologists, one oncology pharmacist and one biostatistician. The evaluation covered different aspects of the model, including the model data, assumptions, methods, analyses and results. The feedback from the panel provided insight into inclusion criteria of AEs into the model and helped verify the modelling approach, analysis plans, plausibility of clinical inputs, duration of treatment effects and outcomes such as projections of OS and PFS. Where consensus was not achieved, the panel provided guidance on the scope of sensitivity analyses. The modelling process, conceptual model and computerized 
Table 4 Base-case model inputs

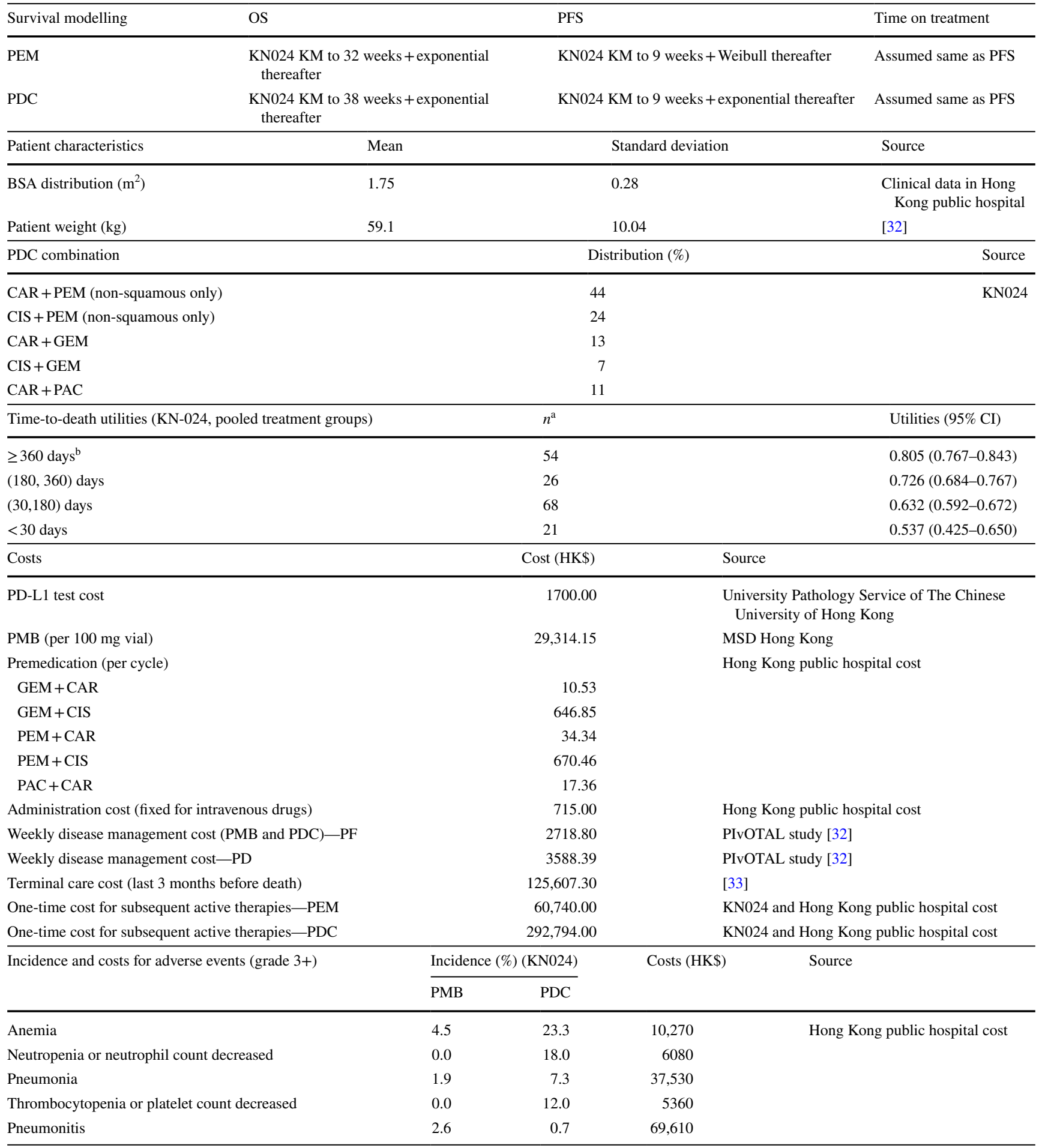

$B S A$ body surface area, $C A R$ carboplatin, $C I$ confidence interval, $C I S$ cisplatin, $G E M$ gemcitabine, $H K \$$ Hong Kong dollar, $K M$ Kaplan-Meier, $M S D$ Merck Sharp \& Dohme, $O S$ overall survival, $P A C$ paclitaxel, $P D$ progressive disease, $P D C$ platinum doublet chemotherapy, $P D-L 1$ programmed death ligand 1, $P E M$ pemetrexed, $P F$ progression free, $P F S$ progression-free survival, $P M B$ pembrolizumab

${ }^{a}$ Number of patients with non-missing EQ-5D index scores

${ }^{b}$ This time-to-death category includes the records of patients whose death dates were observed or censored $\geq 360$ days after the report of EQ-5D scores. Other categories only include the records of patients with an observed death date 
model were evaluated against and satisfied the published AdViSHE criteria for assessment of the validation status of health economic decision models [14, 34].

The extrapolation of OS in the base US model was validated with external observational data. The clinical plausibility of the projected long-term outcomes was also verified by independent clinical experts. The OS curve of platinum doublet chemotherapy was compared with the survival data collected from the Flatiron Health database in patients aged 18 years and newly diagnosed with metastatic NSCLC initiating firstline anticancer therapy (November 2012-January 2015, with follow-up through July 2015) [35]. The OS curve from the Flatiron data matched closely with the projected OS curve of platinum doublet chemotherapy with switching adjustment. The model-projected 5-year survival with platinum doublet chemotherapy with switching adjustment was $3.1 \%$, which is close to the 5-year survival rate of $3.2 \%$ estimated from data for metastatic NSCLC from the National Cancer Institute's Surveillance, Epidemiology, and End Results program [14, 36]. As pembrolizumab is a newly approved indication in the first-line treatment of NSCLC, no observational data were available to validate the OS extrapolation for pembrolizumab [14].

\section{Results}

\subsection{Base-Case Results}

In the base case, the model projected the total average direct treatment costs per patient to be HK\$1147,792
(US\$147,153) in the BTS with pembrolizumab arm and HK\$898,715 (US\$115,220) in the platinum doublet chemotherapy arm, giving an incremental cost of HK $\$ 249,077$ (US\$31,933) over a time horizon of 10 years. The higher overall medical costs associated with the pembrolizumab arm were primarily driven by first-line therapy drug acquisition costs and, to a lesser extent, disease management costs, both being significantly impacted by improved PFS and OS with pembrolizumab use. Conversely, costs for post-discontinuation therapy were lower in the pembrolizumab arm.

It was estimated that patients in the BTS with pembrolizumab arm would survive an average of 0.99 and 1.24 years in the PF and PD states, respectively, leading to a mean survival time of 2.23 years. In contrast, patients in the platinum doublet chemotherapy arm spent less time in the PF state (average 0.55 years) and more time in the PD state (average 1.32 years), for a lower overall mean survival estimate of 1.87 years. Pembrolizumab-treated patients in the BTS approach had an expected QALY and LY gain over platinum doublet chemotherapy of 0.29 and 0.36 years, respectively.

Overall, the model projected ICERs of HK $\$ 865,189$ (US\$110,922) per QALY gained and HK\$697,462 (US\$89,419) per LY gained with pembrolizumab in the BTS approach compared with platinum doublet chemotherapy. Thus, BTS with pembrolizumab was considered a costeffective treatment option for biomarker-identified patients (TPS $\geq 50 \%$ ) with metastatic NSCLC using a willingness-topay (WTP) threshold of HK\$1017,819/QALY (US\$130,490) in Hong Kong. The overall discounted results are presented in Table 5.
Table 5 Cost effectiveness of PD-L1 BTS with pembrolizumab vs. platinum doublet chemotherapy

\begin{tabular}{llll}
\hline & $\begin{array}{l}\text { Platinum doublet } \\
\text { chemotherapy }\end{array}$ & $\begin{array}{l}\text { BTS with pem- } \\
\text { brolizumab }\end{array}$ & $\begin{array}{l}\text { Incremental BTS vs. } \\
\text { platinum doublet chemo- } \\
\text { therapy }\end{array}$ \\
\hline Life-years & 1.87 & 2.23 & 0.36 \\
Progression-free state & 0.55 & 0.99 & 0.44 \\
Progressive disease state & 1.32 & 1.24 & -0.08 \\
QALYs & 1.41 & 1.69 & 0.29 \\
Costs (HK\$) & 898,715 & 1147,792 & 249,077 \\
PD-L1 test cost & 0 & 1700 & 1700 \\
Drug acquisition cost & 147,590 & 420,919 & 273,329 \\
Premedication cost & 947 & 660 & -286 \\
Drug administration cost & 6843 & 8651 & 1808 \\
Disease management cost & 325,350 & 372,312 & 46,962 \\
Post-discontinuation therapy cost & 291,341 & 221,252 & $-70,089$ \\
Terminal care cost & 119,286 & 116,263 & -3023 \\
AE cost & 7357 & 6035 & -1323 \\
ICER (HK\$) & & & 697,462 \\
Cost per LY & & & 865,189 \\
Cost per QALY & & & \\
\hline
\end{tabular}

$A E$ adverse event, BTS biomarker test-and-treat strategy, $H K \$$ Hong Kong dollar, ICER incremental costeffectiveness ratio, $L Y$ life-year, $P D-L 1$ programmed death ligand 1, $Q A L Y$ quality-adjusted life-year 


\subsection{Sensitivity Analyses}

\subsubsection{One-Way Deterministic Sensitivity Analysis}

In the one-way DSA, extrapolation parameters for OS for pembrolizumab and platinum doublet chemotherapy, and PFS for pembrolizumab, were the strongest sources of variation in the ICER. Other influential parameters were health state utilities, costs of post-discontinuation therapy in the platinum doublet chemotherapy arm and weekly disease management costs in the PF state (Fig. 2).

\subsubsection{Probabilistic Sensitivity Analysis}

Figure 3 presents a scatterplot of the 1000 incremental cost and QALY estimates from the Monte Carlo simulation. Based on the CEAC in Fig. 4, the ICER falls below HK\$1000,000/QALY (US\$128,206) with a $58.4 \%$ probability and below HK\$1200,000/QALY (US\$1538,466) with a $68.4 \%$ probability. The CEAC (Fig. 4) showed that, at a WTP threshold of HK\$1,017,819/QALY (US\$130,490), the pembrolizumab BTS had a $59.4 \%$ probability of being cost effective.

\section{Discussion}

The purpose of this cost-effectiveness analysis was to evaluate the costs and benefits of BTS with pembrolizumab compared with platinum doublet chemotherapy in the first-line treatment of metastatic NSCLC in Hong Kong. The BTS approach was projected to yield a gain of 0.29 QALYs at an additional total cost of HK\$249,077 per patient (US\$31,933) compared with platinum doublet chemotherapy, resulting in an ICER of HK\$865,189 (US\$110,922) per QALY gained over a 10-year period. Also, the strategy led to a gain of 0.36
Fig. 2 Tornado diagram for the incremental cost-effectiveness ratio of pembrolizumab vs. platinum doublet chemotherapy
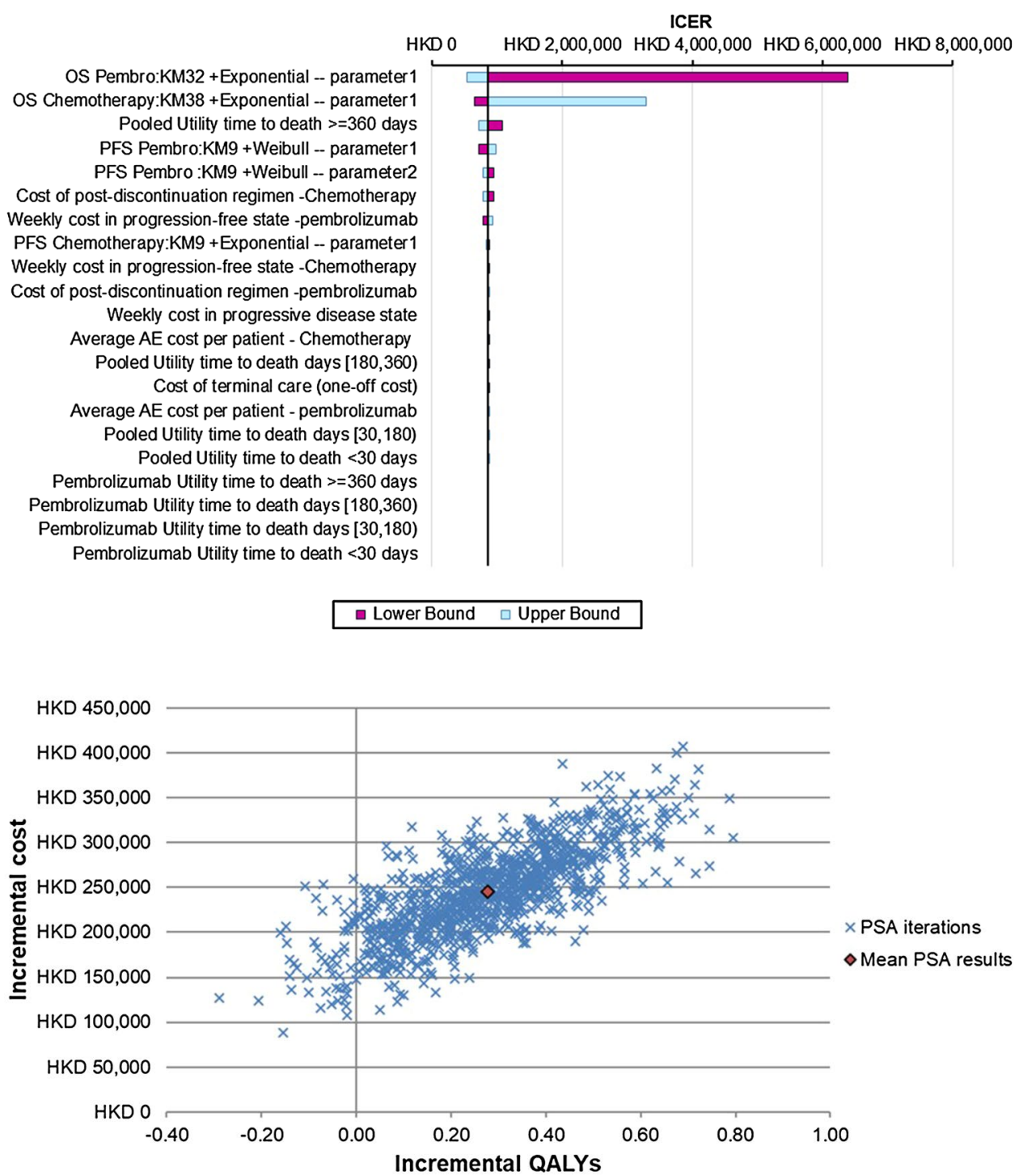

Fig. 3 Probabilistic sensitivity analysis results for pembrolizumab vs. platinum doublet chemotherapy 
Fig. 4 Cost-effectiveness acceptability curve for pembrolizumab vs. platinum doublet chemotherapy

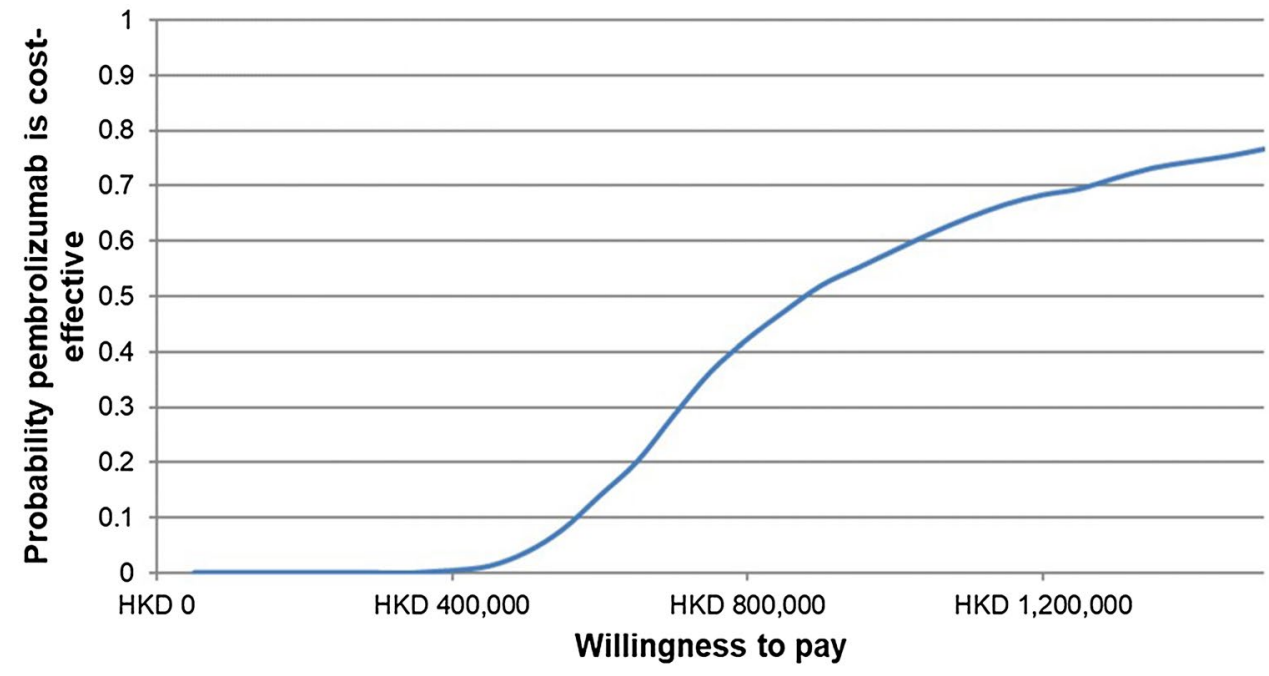

additional years of life, with an incremental cost per LY gain equal to HK\$697,462 (US\$89,406). The key drivers of cost effectiveness were the drug acquisition costs and projections of improved survival. Because the results were sensitive to long-term survival projections, results could differ with the availability of long-term follow-up data [37].

Comprehensive DSA and PSA were performed to assess the robustness of the results. In a scenario analysis, a lower ICER of HK\$859,284 (US\$110,165) was estimated in a comparison of pembrolizumab versus platinum doublet chemotherapy among patients with PD-L1 TPS $\geq 50 \%$. Importantly, when just considering this group of patients who received pembrolizumab, OS was projected to be increased by $63 \%$, and QALYs by $67 \%$, relative to platinum doublet chemotherapy, indicative of a true advancement in patient health outcomes. A previous analysis using the price to patients of pembrolizumab in a Hong Kong public hospital reported an ICER of HK\$598,772 (US\$76,517), which indicated BTS with pembrolizumab was cost effective (file 2 in the ESM).

A key strength of this analysis is its use of efficacy and safety data from a randomized controlled trial directly comparing pembrolizumab and platinum doublet chemotherapy, bolstering the reliability of the incremental cost-effectiveness results. A 2-year stopping rule, as per the KN024 protocol in the model base case, provides certainty to payers and physicians around pembrolizumab treatment duration. The implementation of a time-to-death utility approach enabled more extensive use of the observed clinical trial data from KN024 and the modeling of a fuller patient experience based on observed empirical data.

Disease management costs and HCRU used in the analysis are reflective of real-world Hong Kong clinical practice (Hong Kong public hospital) in metastatic NSCLC. The base case also included the cost of PD-L1 testing in the BTS with pembrolizumab arm. Using PD-L1 IHC assays to screen patients will improve the efficiency of the healthcare system by focusing treatment investment in patients most likely to benefit from pembrolizumab. Also, as suggested by pharmacokinetic modeling, administration of pembrolizumab at a 200-mg fixed dose provides exposure similar to that of the weight-based dosing regimens used in previous studies of pembrolizumab [38].

There are some limitations to this analysis that may require additional research. Local data on clinical efficacy and safety were not available but could have contributed to the OS and PFS specific to patients with NSCLC in Hong Kong. Also, the prevalence of patients with PD-L1 TPS $\geq 50 \%$ was taken from the trial, since no local study of PD-L1 prevalence has been conducted in patients matching those in the KN024 trial from which to evaluate the applicability of the PD-L1 $\geq 50 \%$ prevalence estimate from the KN024 trial to Hong Kong. This study was conducted from a Hong Kong Hospital Authority perspective and is limited to direct lung cancer-related medical costs. Other direct nonmedical costs such as transportation or societal costs (lost productivity or caregiver costs) were not accounted for in the model, so results may not be representative of all payers. As described, we did not evaluate all AEs observed within the trial; only the costs of grade 3-5 AEs affecting $\geq 5 \%$ of patients in both treatment groups were included in the analysis, leading to underestimated AE costs. However, the cost differential that would affect the ICER calculation is not expected to be large given the low incidence rate of the excluded AEs and the impact of AE assumptions generally on the ICER as seen in the DSA. Local valuations for health utility scores were not available, so the analysis used a global valuation approach for utilities and presented DSA results describing the sensitivity of results to varying health utilities. EQ-5D scores were calculated using the 
published EQ-5D-3L value set for a non-Hong Kong general population. The use of an investigator choice comparator (blended comparator) should be interpreted with caution if local clinical practices differ. It is also possible that the real-world duration of treatment with pembrolizumab could differ from that observed in the clinical trial. Also, the duration of follow-up in the clinical trial data used in the model was limited because of the recommendation from the Data Safety Monitoring Board at the second interim analysis that the trial be stopped for efficacy.

Partitioned survival modeling is commonly used to evaluate cost effectiveness in advanced oncology indications, as the approach enables direct use of the trial survival analysis results. A potential for inherent bias in a partitioned survival model has recently been raised by hypothetical modelling and simulated data [39, 40]. However, an empirical analysis using real-world data by Goeree et al. [41] found that the partitioned survival model and the Markov model produced similar ICER estimates in advanced NSCLC, confirming the structural validity of the partitioned survival approach in the current analysis [41]. Still, uncertainty is inherent in the survival projections/extrapolation of PFS and OS to model beyond the trial period. Although several survival curve options were explored in scenario analyses (file 2 in the ESM), additional real-world data will help validate the model in the longer term.

Formal cost-effectiveness decision rules are often not specified by payers. In this situation, as with the present analysis, the WHO recommends using a WTP threshold based on the GDP per capita (three times GDP per capita) [42]. According to the WHO's recommendation, interventions for which the ICER is less than GDP per capita are considered very cost effective, those between one and three times GDP per capita are cost effective, and those greater than three times GDP per capita are not cost effective [43, 44]. The threshold for Hong Kong used in this paper was based on the 2016 per capita GDP (HK\$339,273 [US\$43,497]), as provided by the Hong Kong Census and Statistics Department [45]. Based on this, the cost-effectiveness threshold in Hong Kong Special Administrative Region ranged from HK\$135,790 (US\$17,409) to HK\$224,647 (US\$28,801) (HK\$189,555 [US424,302] to HK\$313,575 [US\$40,202], purchasing power parity adjusted) [42]. While an explicit threshold has not been reported for Hong Kong, the KN024 indication has been publicly reimbursed following the submission of health economic evidence, which may suggest that the threshold lies above the ICERs reported in the presented study. The threshold recommended by the WHO did not reflect the opportunity costs on the healthcare systems. Hence, we also provided the CEAC, which can be used as an alternative threshold, and conclude that the probability of BTS with pembrolizumab being cost effective is around $80 \%$ at a threshold of three times GDP per capita. The probability of cost effectiveness decreases at lower thresholds and increases at higher ones. This evidence of cost effectiveness should be useful in the formal new drug enlistment process of the Hospital Authority Drug Formulary in Hong Kong [46]. However, evaluation using cost-effectiveness analyses does not guarantee affordability. Understanding potential budget impact is also necessary to evaluate the viability of the BTS approach in treating first-line metastatic NSCLC by Hong Kong healthcare providers.

\section{Conclusion}

Despite the higher treatment costs of pembrolizumab, this analysis demonstrated that BTS with pembrolizumab was associated with significantly longer OS and PFS, a lower frequency of treatment-related AEs and more QALYs. Taken together, this economic analysis suggests that a BTS to identify a subset of patients with NSCLC with PD-L1 TPS $\geq 50 \%$ for treatment with pembrolizumab in the first-line setting can be considered cost effective in Hong Kong. These health benefits will accrue at an incremental cost of HK\$865,189 (US\$110,922) per QALY gained with a BTS approach versus investigator's choice platinum doublet chemotherapy. This is projected to lead to health gains to society at a reasonable level of medical expenditure based on the WHO criterion.

Acknowledgements The authors thank Dr. John Cook (Complete HEOR Solutions LLC, 1120 Welsh Road, Suite 205, North Wales, PA, 19454 USA) for his help and insight in drafting the manuscript, Dr. Sujata Basu (Complete HEOR Solutions LLC, 1120 Welsh Road, Suite 205, North Wales, PA, 19454 USA) for her contribution to the research and analysis and Adeline Law for her help with the analysis.

Author contributions Drs. HL and ST contributed to the planning of the study, acquisition of data, and interpretation of results and critically reviewed/revised the manuscript. Drs. CW, LL, RI and SC interpreted the results and critically reviewed/revised the manuscript. Dr. $\mathrm{MH}$ contributed to the planning of the study, acquisition and analysis of the data, and interpretation of results and critically reviewed/revised the manuscript. Drs. JP and PD contributed to the planning of the study and interpretation of results and critically reviewed/revised the manuscript. ML and DH contributed to the acquisition of data and interpretation of results and critically reviewed/revised the manuscript. AR and MA adapted the model, conducted the analyses, interpreted the results and wrote the manuscript. All authors read and approved the final manuscript.

\section{Compliance with Ethical Standards}

Availability of data and material All data generated or analyzed during this study are included in this published article (and its supplementary files).

Funding Funding was provided by Merck Sharp \& Dohme Corp., a subsidiary of Merck \& Co., Inc., Kenilworth, NJ, USA. 
Conflict of Interest RI, MH, JP and SC are employees of Merck \& Co., Inc., Kenilworth, NJ, USA. ST is an employee of MSD Asia Pacific, Singapore. ML and DH are employees of Merck Sharp \& Dohme (Hong Kong), Hong Kong. CW and LL have no conflicts of interest that are directly relevant to the content of this article. HL has received grants from MSD outside the submitted work. CHEORS received funds from Merck \& Co. Inc., during the conduct of the study; PD, AR and MA are employees of CHEORS and received personal fees from Merck \& Co. Inc., outside the submitted work.

Open Access This article is distributed under the terms of the Creative Commons Attribution-NonCommercial 4.0 International License (http://creativecommons.org/licenses/by-nc/4.0/), which permits any noncommercial use, distribution, and reproduction in any medium, provided you give appropriate credit to the original author(s) and the source, provide a link to the Creative Commons license, and indicate if changes were made.

\section{References}

1. Le Chevalier T. Adjuvant chemotherapy for resectable non-small-cell lung cancer: where is it going? Ann Oncol. 2010;21(suppl_7):vii196-8.

2. Estimated cancer incidence, mortality and prevelance worldwide in 2012-lung cancer: GLOBOCAN. 2012. http://globocan.iarc. fr/Pages/fact_sheets_cancer.aspx. Accessed 17 Nov 2017.

3. Hong Kong Cancer Registry: Hong Kong Hospital Authority. http://www3.ha.org.hk/cancereg/. Accessed 20 Nov 2017.

4. Lung Cancer. The Government of Hong Kong Special Administrative Region. https://www.chp.gov.hk/en/healthtopics/conte nt/25/49.html. Accessed 20 Nov 2017.

5. What are the key statistics about lung cancer? https://www.cance r.org/cancer/non-small-cell-lung-cancer/about/key-statistics.html. Accessed 20 Nov 2017.

6. Lung cancer incidence statistics. 2016. https://seer.cancer.gov/ csr/1975_2016/. Accessed 20 Nov 2017.

7. Gerber DESJ. Maintenance chemotherapy for advanced nonsmall-cell lung cancer: new life for an old idea. J Clin Oncol. 2013;31(8):1009.

8. Reck M PS, Reinmuth N, De Ruysscher D, Kerr KM, Peters S. Metastatic non-small-cell lung cancer (NSCLC): ESMO Clinical Practice Guidelines for diagnosis, treatment and follow-up. Ann Oncol. 2014;25(suppl_3):iii27-iii39.

9. NB. L. Treatment paradigms for patients with metastatic nonsmall-cell lung cancer: first-, second-, and third-line. Curr Oncol. 2012;19(Suppl 1):S52.

10. Merck Oncology. Merck Sharp \& Dohme Corp., a subsidiary of Merck \& Co., Inc.; 2017. https://www.merck.com/index.html. Accessed 24 Nov 2017.

11. US Department of Health and Human Services. https://www.fda. gov/drugs/resources-information-approved-drugs/pembrolizu mab-keytruda-checkpoint-inhibitor. Accessed 24 Nov 2017.

12. Garon EBRN, Hui R, Leighl N, Balmanoukian AS, Eder JP, Patnaik A, Aggarwal C, Gubens M, Horn L, Carcereny E. Pembrolizumab for the treatment of non-small-cell lung cancer. N Engl J Med. 2015;372(21):2018-28.

13. Reck M R-AD, Robinson AG, Hui R, Csőszi T, Fülöp A, Gottfried M, Peled N, Tafreshi A, Cuffe S, O’Brien M. Pembrolizumab versus chemotherapy for PD-L1-positive non-small-cell lung cancer. N Engl J Med. 2016;375(19):1823-33.

14. Huang M LY, Pellissier J, Burke T, Liu FX, Xu R, Velcheti V. Cost effectiveness of pembrolizumab vs. Standard-of-care chemotherapy as first-line treatment for metastatic NSCLC that expresses high levels of PD-L1 in the United States. PharmacoEconomics. 2017;35(8):831-44.

15. Owen LMA, Fischer A, Ellis S, Hoy A, Kelly MP. The costeffectiveness of public health interventions. J Public Health. 2011;34(1):37-45.

16. Partitioned Survival Model. York; York Health Economics Consortium; 2016. https://www.yhec.co.uk/glossary/partitioned-survi val-model/. Accessed 17 July 2017.

17. National Institute for Health and Clinical Excellence (NICE). NICE guide to methods of technology appraisal, UK, 2013. https ://www.nice.org.uk/process/pmg9/chapter/foreword. Accessed 13 July 2017.

18. Canadian Agency for Drugs and Technologies in Health. Guidelines for the economic evaluation of health technologies: Canada. 3rd ed. Ottawa: Canadian Agency for Drugs and Technologies in Health; 2006. p. 2006.

19. Annual report on consumer price index, Census and Statistics Department Hong Kong. https://www.censtatd.gov.hk/hkstat/sub/ sp270.jsp?productCode=B1060002. Accessed 15 May 2016.

20. Sorensen SFZW, Dolled-Filhart M, Georgsen JB, Wang Z, Emancipator K, Wu D, Busch-Sørensen M, Meldgaard P, Hager H. PD-L1 expression and survival among patients with advanced non-small cell lung cancer treated with chemotherapy. Transl Oncol. 2016;9(1):64-9.

21. Sun JMZW, Choi YL, Choi SJ, Kim SE, Wang Z, Dolled-Filhart M, Emancipator K, Wu D, Weiner R, Frisman D. Prognostic significance of PD-L1 in patients with non-small cell lung cancer: a large cohort study of surgically resected cases. J Thorac Oncol. 2016;11(7):1003-11.

22. KEYTRUDA: Prescribing Information. Food and Drug Administration; 2016. https://www.accessdata.fda.gov/drugsatfda_docs/ label/2016/125514s012lbl.pdf. Accessed 15 Nov 2016.

23. Latimer N. NICE DSU Technical Support Document 14: survival analysis for economic evaluations alongside clinical trials Extrapolation with patient-level data. Sheffield: Report by the Decision Support Unit, 2011; 2013.

24. NICE DSU Technical Support Document 16: Adjusting survival time estimates in the presence of treatment switching. London: NICE. http://www.nice.dsu.org.uk/TSD16_Treatment_Switching. pdf. Accessed 17 July 2017.

25. Latimer NR. Survival analysis for economic evaluations alongside clinical trials-extrapolation with patient-level data: inconsistencies, limitations, and a practical guide. Med Decis Making. 2013;33(6):743-54.

26. Chow GC. Chow tests. 2011. https://en.wikipedia.org/wiki/Chow_ test. Accessed 12 Dec 2017.

27. Chow GC. Tests of equality between sets of coefficients in two linear regressions. Econometrica. 1960;28(3):591-605.

28. Huang M PJ, Kong F, editor. A Trial-Based Euroqol Eq-5d Health Utility Analysis in Patients with Previously Untreated Metastatic NSCLC. ISPOR 22nd annual international meeting; Boston, MA; 2017.

29. Shaw JW JJ, Coons SJ. US valuation of the EQ-5D health states: development and testing of the D1 valuation model. Med Care. 2005:203-20.

30. Dolan P. Modeling valuations for EuroQol health states. Med Care. 1997:1095-108.

31. Greiner WWT, Nieuwenhuizen M, Oppe S, Badia X, Busschbach J, Buxton M, Dolan P, Kind P, Krabbe P, Ohinmaa A. A single European currency for EQ-5D health states. Eur J Health Econ HEPAC. 2003;4(3):222-31.

32. Data on file. Merck Kenilworth NZ; PIVOTAL study: Global treatment patterns, resource utilization and biomarker testing of advanced non-small cell lung cancer. 
33. Wong IO KK, Cowling BJ, Lam CL, Leung GM. Cost effectiveness of mammography screening for Chinese women. Cancer: Interdiscip Int J Am Cancer Soc. 2007;110(4):885-95.

34. Vemer PRI, Van Voorn GA, Al MJ, Feenstra TL. AdViSHE: a validation-assessment tool of health-economic models for decision makers and model users. Pharmacoeconomics. 2016;34(4):349-61.

35. Flatiron Health. https://flatiron.com/life-sciences. Accessed 18 Feb 2018.

36. National Cancer Institute. National Cancer Institute SEER Data, 1973-2013. https://seer.cancer.gov/data/. Accessed 17 Oct 2017.

37. Updated Analysis of KEYNOTE-024: Pembrolizumab vs Platinum-Based Chemotherapy for Advanced NSCLC With PD-L1 TPS $\geq 50 \%$. 18th World Conference on Lung Cancer (WCLC); 2017; Yokohama, Japan. http://wclc2017.iaslc.org/. Accessed 17 Dec 2017.

38. Freshwater T, Kondic A, Ahamadi M, Li CH, de Greef R, de Alwis D, Stone JA. Evaluation of dosing strategy for pembrolizumab for oncology indications. J Immunother Cancer. 2017;5(1):43.

39. Beca JHJ. Exploring the impact of structural uncertainty in partitioned survival models for oncology. Value Health. 2014;17(3):A205-6.

40. Coyle DCK. The inherent bias from using partitioned survival models in economic evaluation. Value Health. 2014;17(3):A194.
41. Goeree RVJ, Goeree J, Penrod JR, Orsini L, Tahami Monfared AA. Economic evaluation of nivolumab for the treatment of second-line advanced squamous NSCLC in Canada: a comparison of modeling approaches to estimate and extrapolate survival outcomes. J Med Econ. 2016;19(6):630-44.

42. Woods BRP, Sculpher M, Claxton K. Country-level cost-effectiveness thresholds: initial estimates and the need for further research. Value Health. 2016;19(8):929-35.

43. Macroeconomics and Health. Investing in health for economic development. Prepared for the World Health Organization.: Commission on Macroeconomics and Health; 2001.

44. LA Robinson HJ, Chang AY, Resch S. Understanding and improving the one and three times GDP per capita cost-effectiveness thresholds. Health Policy Plann. 2016;32(1):141-5.

45. Census and Statistics Department, Hong Kong. 2005. https://www. censtatd.gov.hk/hkstat/sub/sp250.jsp?tableID=030\&ID=0\&produ ctType $=8$. Accessed 15 Mar 2018.

46. Wong CKWO, Cheung BM. Towards a transparent, credible, evidence-based decision-making process of new drug listing on the hong kong hospital authority drug formulary: challenges and suggestions. Appl Health Econ Health Policy. 2018;16(1):5-14. 